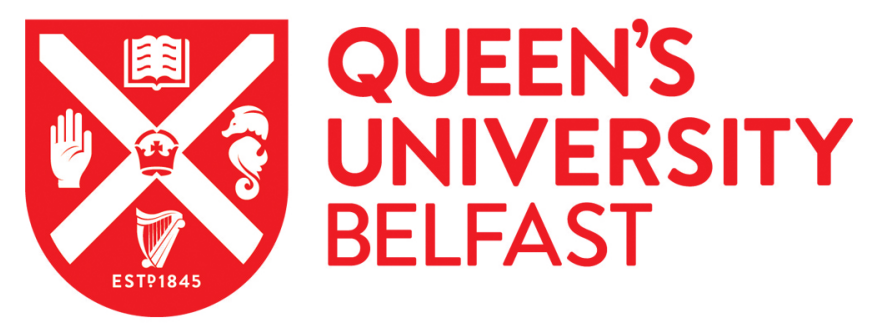

\title{
Acceptance and Action Questionnaire - II Confirmatory factor analysis and measurement invariance between Non-Hispanic White and Hispanic/Latinx undergraduates
}

Edwards, K. A., \& Vowles, K. E. (2020). Acceptance and Action Questionnaire - II Confirmatory factor analysis and measurement invariance between Non-Hispanic White and Hispanic/Latinx undergraduates. Journal of Contextual Behavioral Science, 17, 32-38. https://doi.org/10.1016/j.jcbs.2020.05.003

Published in:

Journal of Contextual Behavioral Science

Document Version:

Peer reviewed version

Queen's University Belfast - Research Portal:

Link to publication record in Queen's University Belfast Research Portal

\begin{abstract}
Publisher rights
Copyright 2020 Elsevier.

This manuscript is distributed under a Creative Commons Attribution-NonCommercial-NoDerivs License

(https://creativecommons.org/licenses/by-nc-nd/4.0/), which permits distribution and reproduction for non-commercial purposes, provided the author and source are cited.

\section{General rights}

Copyright for the publications made accessible via the Queen's University Belfast Research Portal is retained by the author(s) and / or other copyright owners and it is a condition of accessing these publications that users recognise and abide by the legal requirements associated with these rights.
\end{abstract}

\section{Take down policy}

The Research Portal is Queen's institutional repository that provides access to Queen's research output. Every effort has been made to ensure that content in the Research Portal does not infringe any person's rights, or applicable UK laws. If you discover content in the Research Portal that you believe breaches copyright or violates any law, please contact openaccess@qub.ac.uk. 
Acceptance and Action Questionnaire - II: Confirmatory Factor Analysis and Measurement Invariance Between Non-Hispanic White and Hispanic/Latinx Undergraduates

Karlyn A. Edwards ${ }^{1 *} \&$ Kevin E. Vowles ${ }^{12}$

${ }^{1}$ Department of Psychology, University of New Mexico, Albuquerque, New Mexico, USA

${ }^{2}$ School of Psychology, Queen's University Belfast, Belfast, UK

16 April 2020

${ }^{*}$ Corresponding Author: Karlyn A. Edwards, M.S., Department of Psychology, University of New Mexico, MSC03-2220 Logan Hall, Albuquerque, NM, 87131-0001, kaedwards@unm.edu 


\begin{abstract}
The AAQ-II is a widely used measure of experiential avoidance, which is defined as an unwillingness to experience aversive private events (i.e. body sensations, emotions, thoughts). Previous studies examining the AAQ-II's psychometric properties have found broad support for a one factor model. However, only one study has examined the factor structure among Hispanic/Latinx (H/L) individuals, and no study has tested for measurement invariance in this group. Measurement invariance is an important consideration, as it is a requirement for score comparisons between distinct samples. Therefore, the current study sought to test a one factor model and measurement invariance in a large sample of $\mathrm{H} / \mathrm{L}$ and Non-Hispanic White (NHW) individuals attending a large university in the Southwest of the United States. Confirmatory factor analyses found a one factor structure to fit adequately in the entire sample, as well as within the H/L and NHW groups separately. Tests of measurement invariance found the one factor structure to be invariant across racial/ethnic groups. Correlational analyses found higher AAQ-II scores to be moderately associated with worse social functioning, depression, anxiety, stress, and self-compassion, and weakly associated with worse physical functioning and dispositional mindfulness. These findings suggest that the one factor model is appropriate and can be used for score comparison between $\mathrm{H} / \mathrm{L}$ and NHW individuals.
\end{abstract}

Keywords: Experiential avoidance, Acceptance and Action Questionnaire - II (AAQ-II), Acceptance and Commitment Therapy (ACT), Hispanic/Latinx, Non-Hispanic White 


\section{Introduction}

Experiential avoidance refers to a process by which humans work to avoid, alter, or suppress difficult private experiences. For example, individuals with chronic pain may avoid certain body movements to avoid the experience of pain, or individuals struggling with significant anxiety may engage in problematic substance use to avoid feeling anxious (Hayes, Wilson, Gifford, Follette, \& Strosahl, 1996). It is conceptualized as a transdiagnostic process across a number of conditions, including substance use, anxiety, depression, eating disorders, and a variety of chronic physical health conditions, such as chronic pain (Levin et al., 2013; Shorey et al., 2017; Spinhoven, Drost, de Rooij, van Hemert, \& Penninx, 2014). It is also theorized to be a key driver of poorer quality of life among non-clinical samples (Kashdan, Barrios, Forsyth, \& Steger, 2006; Stockton et al., 2018). While many behavioral treatments have historically recognized the harmful effects of experiential avoidance, acceptance and mindfulness-based interventions target this process directly with adequate effectiveness. Of those, Acceptance and Commitment Therapy (ACT) is designed specifically to decrease experiential avoidance and facilitate greater acceptance of difficult experiences in the service of engaging in personally valued activities consistently over the longer term (Hayes, Pistorello, \& Levin, 2012; Hayes et al., 1996; McCracken \& Vowles, 2014; Rolffs, Rogge, \& Wilson, 2016).

The most widely used self-report measure of experiential avoidance is the Acceptance and Action Questionnaire (AAQ-II; Bond et al., 2011), which includes seven items that create a single factor. The initial validation study examined the one factor structure and measurement invariance between three large clinical and non-clinical samples, which included undergraduates, patients receiving outpatient substance use treatment, and employees of a large corporation. The one factor structure was supported and found to be invariant across all three samples. In addition, 
the AAQ-II had adequate internal consistency (Cronbach's alpha $=.78-.87)$ and three and twelve month test-rest reliability (.81 and .79, respectively; Bond et al., 2011).

In subsequent studies, the AAQ-II's psychometric properties have been tested among multiple large and nationally diverse samples. Most notably, it was tested within a large international sample $(N=2,170$; Monestès et al., 2018) comprising five European countries. Consistent with previous findings, a one factor structure was supported and invariant across all samples. Further, correlations with measures of emotional functioning supported convergent validity, such that a higher AAQ-II score, or higher experiential avoidance, was associated with worse depression, dispositional mindfulness, and more frequent engagement in thought suppression (Monestès et al., 2018). The psychometric properties of the AAQ-II have also been examined and validated in a number of other diverse samples, including clinical and non-clinical samples in Colombia, China, Portugal, Greece, and Hungary (Eisenbeck \& Szabó-Bartha, 2018; Fledderus, Voshaar, Klooster, \& Bohlmeijer, 2012; Karekla \& Michaelides, 2017; Ruiz et al., 2016; Zhang, Chung, Si, \& Liu, 2014).

While the AAQ-II's psychometric properties have been supported in previous work, the samples have primarily consisted of Non-Hispanic White (NHW) or White European individuals. Assessment of the AAQ-II's psychometric properties among racially/ethnically diverse samples is particularly important given that cultural beliefs and acculturative stress have been shown to impact experiential avoidance (Su, Wei, \& Tsai, 2014; Zvolensky, Jardin, Garey, Robles, \& Sharp, 2016). Specifically, a recent study found that among Hispanic/Latinx (H/L) individuals that were high on experiential avoidance also experienced significantly more acculturative stress as compared to those who were lower on experiential avoidance. Further, higher acculturative stress and experiential avoidance were both found to be uniquely related to 
worse suicidal ideation, social anxiety, and arousal symptoms (Zvolensky et al., 2016). To date, only one study has examined the factor structure of the AAQ-II among $\mathrm{H} / \mathrm{L}$ individuals, which supported a one factor model, although this study did not conduct tests of measurement invariance (Flynn, Berkout, \& Bordieri, 2016). It is particularly important to test measurement invariance of the AAQ-II among $\mathrm{H} / \mathrm{L}$ individuals given that experiential avoidance is theorized to be a transdiagnostic process, and measurement non-invariance would suggest the need to revise the measure in order to accurately assess experiential avoidance among $\mathrm{H} / \mathrm{L}$ individuals. It is also important to note that recent work has carefully examined the discriminant validity of the AAQ-II in terms of its ability to discern the presence of distress and one's response to distress (i.e., experiential avoidance). Both Wolgast (2014) and Tyndall et al. (2019) found that the AAQ-II was more strongly correlated with measures of distress than responses to distress. The latter study, however, also supported the convergent validity between the AAQ and the Brief Experiential Avoidance Questionnaire, a measure of distress responding. A third study (Ong, Pierce, Woods, Twohig, \& Levin, 2018) examined item level responses to the AAQ-II and found that items did not show differential functioning based on symptom severity or clinical presentation. The authors concluded that the AAQ-II is still a useful tool to measure experiential avoidance, however, particularly given its current level of supportive evidence. Together, while these studies raise issues with the AAQ-II and its ability to discriminate between distress and distress responding, it remains a widely used measure with established psychometric properties and examination of its performance in diverse samples is highly important.

The current study sought to extend upon previous work by examining the fit of the one factor model of the AAQ-II in a large sample of NHW and H/L undergraduates and to test measurement invariance between these two ethnic groups. Given the previous study on H/L 
individuals (Flynn et al., 2016), it was hypothesized that the one factor model would be supported in the NHW and H/L samples. It was also hypothesized that the AAQ-II factor structure would be invariant across the NHW and H/L ethnic groups. Finally, it was hypothesized that higher AAQ-II scores would be significantly correlated with poorer functioning across eight measures, including both measures of distress (i.e., anxiety, depression, stress) and responses to distress (i.e., polysubstance use in the past month, physical and social functioning, self-compassion, and mindfulness).

\section{Methods}

\section{Participants}

The current study is a secondary data analysis of a previous study examining social norms of substance use among college undergraduates at a large Southwestern University in the United States (Edwards, Witkiewitz, \& Vowles, 2019). Eligibility criteria required that participants were $\geq 18$ years old, enrolled as an undergraduate student, and able to read and write English. All participants were recruited from undergraduate psychology courses through an online research system. Data were collected from a total of 2,243 participants. Data for 763 (34.0\%) individuals were excluded from these analyses for the following reasons: 224 due to performing below an $80 \%$ accuracy on the validity check questions, 158 due to being older than the typical college age range $(18-26)$, and 56 due to excessive (i.e., $\geq 75 \%)$ missing responses. The final 325 participants with excluded data selected a race/ethnicity other than NHW or H/L. Therefore, total sample size was 1,509 for the present analyses.

\section{Procedure}

Students interested in completing the survey for class credit were provided with a URL link to the informed consent hosted on an online survey platform. To enroll, participants were 
required to read the informed consent and confirm that they met the stated eligibility criteria prior to accessing the study questionnaires. Participants were asked to complete a survey assessing personal and peer perceptions of substance use, multiple measures of functioning, and demographic characteristics. Five validity check questions were randomly placed throughout the survey and participants were required to accurately complete at least four of these questions. Responses for the current study were submitted between June 2017 and March 2019. All study procedures were reviewed and approved by the university Institutional Review Board.

\section{Measures}

Demographic Information. Demographics collected included self-reported gender, age, race/ethnicity, class standing, family income, grade point average, and employment status.

Acceptance and Action Questionnaire - II. As noted, the AAQ-II contains seven items to measure experiential avoidance. Participants are asked to rate how true each of the seven statements were for them, and responses are measured on a 7-point Likert-type scale range from 1 'never true' to 7 'always true'. Total scores are calculated by summing all seven items and range from 7 to 49, with higher scores indicating greater experiential avoidance. Sample items include 'I'm afraid of my feelings', 'Emotions cause problems in my life', and 'Worries get in the way of my success'.

Psychological Processes and Outcomes. Eight important markers of physical and psychological health were used in current analyses. Polysubstance use was assessed by asking participants to indicate how many categories of substances they had used recreationally in the past 30 days. Categories included alcohol, cannabis, opioids, heroin, stimulants, sedatives, and psychedelics. For those substances that may be prescribed, recreational use was defined as use without a prescription or using more than prescribed. Physical and social functioning was 
assessed using the Short-Form 36 (SF-36), which is a 36-item measure that asks participants to rate their engagement in physical and social activity in the past four weeks. The measure yields two subscales, with higher scores indicating worse physical and social disability (Brazier et al., 1992; DeBerard \& Masters, 2014). The SF-36 has been found to have adequate construct validity within a H/L primary care patient sample (Katerndahl, Amodei, Larme, \& Palmer, 2002). Depression, anxiety, and overall stress was assessed using the Depression, Anxiety, and Stress Scale (DASS-21), which is a 21-item measure that asks participants to rate their depression, anxiety, and stress symptom severity in the past week. The measure yields three subscales with higher scores indicating worse depression, anxiety, and stress. The DASS-21 does have support for adequate validity and reliability among a large college student sample as well as a large H/L college student sample (Camacho, Cordero, \& Perkins, 2016; Osman et al., 2012).

Self-compassion was assessed using the Self-Compassion Scale (SCS), which is a 24item measure that asks participants to rate how they act towards themselves during difficult and challenging situations. For example, items include, 'When times are difficult, I tend to be tough on myself' and 'I try to be loving towards myself when I'm feeling emotional pain'. The measure yields a total score, with higher scores indicating higher self-compassion. Further, the scale does have support for strong validity and reliability across college students as well as among multiple racial/ethnic groups (Neff, 2003; Neff et al., 2018; Neff, Whittaker, \& Karl, 2017). Lastly, mindfulness was assessed using the Mindful Attention Awareness Scale (MAAS), which is a 15 -item measure that asks participants to rate how often they use mindfulness techniques in their day to day experience. For example, items include, 'I find it difficult to stay focused on what is happening in the present' and 'I find myself listening to someone with one ear, and doing something else at the same time'. The measure yields a mean total score, with 
higher scores indicating higher dispositional mindfulness (Brown \& Ryan, 2003; Osman et al., 2016).

\section{Data Analysis Plan}

All analyses were conducted using Mplus Version 8.2 (Muthén \& Muthén, 2017). The current sample size for each group met standard recommendations stating at least 200 per group (Kline, 2015). Data were screened for non-normality and outliers, and the normality assumption was violated. Therefore, weighted least square (WLSMV) estimation was used as it is robust to violations of normality and is less biased with ordinal data ( $\mathrm{Li}, 2016)$. In order to evaluate any demographic differences between the two ethnic groups, $t$ - test and chi-square analyses were carried out initially. Next, an independent sample $t$ - test was used to test if there were significant differences in total AAQ-II scores between ethnic groups.

The factor structure of the AAQ-II was analyzed using Confirmatory Factor Analysis (CFA) with unit loading identification. The standardization method (i.e. fixing the variance of the latent factor to one) was used for model identification, and questionnaire items were treated as ordinal data. Model fit was examined using the model chi-square test $\left(\chi^{2}, p>.05\right)$, Comparative Fit Index (CFI; cut off $\geq 0.90)$, Tucker-Lewis Index (TLI; cut off $\geq 0.95$ ), root mean square error of approximation (RMSEA; cut off $\leq 0.08)$, and standardized root mean square residual (SRMR; cut off $\leq .08 ;$ Kline, 2015). For all confirmatory factor analyses, a one factor model with all seven items loading onto one latent factor was tested. Residual correlation between items 1 and 4 were included in each model, given that the original psychometric paper accounted for this path due to the items similar wording (Bond et al., 2011). In addition, including residual correlation paths can improve potential bias of the latent factor (Cole, Ciesla, \& Steiger, 2007). Items 1 and 4 were 'My painful experiences and memories make it difficult for 
me to live a life that I would value' and 'My painful memories prevent me from having a fulfilling life', respectively. Initially, a one factor model was tested within the total sample. Next, the same model was tested within the NHW and H/L groups separately.

To test measurement invariance, successively restrictive models were fit to the data (Chen, Sousa, \& West, 2014). First, a configural model was fit to the data, which examined whether the factor structure of the AAQ-II was equivalent between racial/ethnic groups. Second, a metric model was fit to the data, which examined whether the factor loadings were equivalent between racial/ethnic groups. Lastly, a scalar model was fit to the data, which examined whether both the factor loadings and the item thresholds were equivalent between racial/ethnic groups. Lastly, to test whether the AAQ-II was associated with eight psychological processes and functioning outcomes, correlations were calculated between the AAQ-II and polysubstance use in the past month, physical and social functioning, depression, anxiety, stress, self-compassion, and dispositional mindfulness. These were calculated for the total sample, as well as among the NHW and H/L groups separately. To test whether the correlations between the racial/ethnic groups were statistically different from each other, the difference between correlation coefficients were first converted into $z$ - scores, and statistical significance was assessed using a two-tailed test at an alpha level of .05.

\section{Results}

\section{Descriptive statistics}

Table 1 shows demographic characteristics for the total sample and each ethnic group in the study. The final sample was primarily women (71\%), H/L (58\%), and freshmen in class standing (52\%). An independent sample $t$-test was used to test differences in age between the NHW and H/L groups. Levene's test for equality of variances was violated $(F=10.05, p=.002)$, 
therefore equal variances were not assumed, which found no differences in age between NHW and $\mathrm{H} / \mathrm{L}$ students $[t(1,257)=1.75, p=.08]$. Chi-square analyses examining differences in gender and class standing between NHW and H/L students indicated no differences between groups [Gender: $\chi^{2}(1)=3.36, p=.07$, Class Standing: $\left.\chi^{2}(3)=1.03, p=.80\right]$. A chi-square analysis examining differences in employment status was significant $\left[\chi^{2}(2)=19.73, p<.001\right.$, such that a higher proportion of $\mathrm{H} / \mathrm{L}$ students were working part-time or full-time in comparison to NHW students. Additionally, a chi-square analysis examining differences in family income was significant $\left[\chi^{2}(4)=83.93, p<.001\right]$, such that a higher proportion of H/L students reported their family income to be $<\$ 25,000$, and $\$ 26,000$ - $\$ 50,000$, and a higher proportion of NHW students reported their family income to be $\geq \$ 100,000$. There were no differences observed between NHW $(M=20.51, S D=9.59)$ and H/L students $(M=20.28, S D=9.76)$ on the total score of the AAQ-II $[t(1,491)=.44, p=.66]$. Internal consistency of the AAQ-II in both the NHW and H/L groups indicated good reliability (Cronbach's Alpha for both groups $=.90$ ).

\section{Confirmatory factor analyses}

A series of confirmatory factor models were tested for fit within the whole sample, followed by NHW and H/L undergraduates separately. A summary of fit statistics for all models can be found in Table 2. Among the whole sample, the one factor model was found to have adequate fit. The CFI, TLI, and SRMR indicated good fit, however the model chi-square test was significant, and the RMSEA was marginally outside the specified range for good fit. In addition, all items significantly loaded onto one latent factor, and the residual correlation between Item 1 and 4 was significant (see Figure 1). These findings replicate previous work supporting the factor structure of the AAQ-II. Given that the model was sufficient in the full sample, it was next tested separately within each ethnic group. 
Among the NHW group, the one factor model was also found to have adequate fit. The CFI, TLI, and SRMR indicated good fit. Similar to the whole sample model fit, the model chisquare test was significant and the RMSEA was marginally outside the specific range for good fit. All items significantly loaded onto one latent factor and the residual correlation path was significant as well. Among the H/L group, the one factor model was also found to have adequate fit. Similar to the two previous models, the CFI, TLI, and SRMR indicated good fit, however the model chi-square test was significant and the RMSEA was marginally outside the specific range for good fit. All items significantly loaded onto the one latent factor and the residual correlation path was significant.

\section{Measurement invariance}

A test of configural invariance indicated that the one factor model fit the data well in both racial/ethnic groups. A similar pattern of fit indices was observed, such that the CFI, TLI, and SRMR indicated good fit, while the model chi-square test was significant and the RMSEA was marginally outside of the specified range for good fit. This suggests that the factor structure of the AAQ-II is the same within each racial/ethnic group. Next, a metric model was fit to the data, and a chi-square difference test was used to compare the fit of the metric and configural invariance models. This difference test was not significant $\left[\chi^{2}(7)=4.73, p=.69\right]$, indicating that the metric model had similar fit to the configural model. This suggests that the item factor loadings onto the latent factor are similar across the NHW and H/L groups. Next, a scalar model was fit to the data, and a chi-square difference test was used to compare the fit of the metric and scalar invariance models. This difference test was not significant $\left[\chi^{2}(7)=40.10, p=.56\right]$, indicating that the scalar model had similar fit as the metric model. This finding suggests that the item thresholds are invariant across the NHW and H/L groups. In addition, change in CFI across 
all models was minimal, supporting measurement invariance across both racial/ethnic groups. Taken together, the one factor measurement model for the AAQ-II was found to be invariant and equivalent across both NHW and H/L undergraduates.

\section{Correlations with Psychological Processes and Functioning Outcomes}

Correlations between AAQ-II scores and eight psychological processes and functioning outcomes among the whole sample and each racial/ethnic group separately can be found in Table 3. Overall, AAQ-II was associated with the eight measures in the hypothesized directions for the whole sample as well as within each racial/ethnic group. Specifically, higher AAQ-II scores had a significant moderate association with worse anxiety, depression, and stress symptom severity, as well as worse social functioning and self-compassion, which was consistent across racial/ethnic groups. Higher AAQ-II scores also had a significant, although weak, association with physical functioning and dispositional mindfulness across racial/ethnic groups. Polysubstance use in the past month was not significantly associated with AAQ-II scores among $\mathrm{H} / \mathrm{L}$ individuals, while it was significantly associated with AAQ-II scores among NHW individuals. A difference test between correlational coefficients between racial/ethnic groups indicated a significant difference $(z=2.43, p=.015)$. There were no other significant differences between correlational coefficients between racial/ethnic groups, with the exception of anxiety wherein higher AAQ-II scores was more strongly associated with worse anxiety among NHW individuals as compared to $\mathrm{H} / \mathrm{L}$ individuals $(z=1.98, p=.047)$.

\section{Discussion}

The key findings from this study indicate that a one factor structure of the AAQ-II was supported in a sample of NHW and H/L undergraduates, and that the measure was found to be invariant between ethnic groups. The present findings extend the work of Flynn, Berkout, \& 
Bordieri (2016) by confirming the factor structure of the AAQ-II in a sample of NHW and H/L undergraduates, in addition to providing support for comparing AAQ-II scores between racial/ethnic groups. The present findings are also in line with the growing literature providing support for the AAQ-II's robust psychometric properties in the assessment of experiential avoidance (Bond et al., 2011; Fledderus et al., 2012; Monestès et al., 2018). Further, across racial/ethnic groups, higher AAQ-II scores were significant and moderately associated with worse social functioning, depression, anxiety, stress, and self-compassion. Higher scores were also significantly, although weakly, associated with physical functioning and dispositional mindfulness. Contrary to previous findings, higher AAQ-II scores were not significantly associated with polysubstance use in the past month among $\mathrm{H} / \mathrm{L}$ individuals, while a significant, but weak association, was observed among NHW individuals.

Experiential avoidance, described as unwillingness to experience and avoidance of distressing private experiences (i.e. body sensations, emotions, and thoughts), is posited to be a transdiagnostic process that contributes to suffering across a number of domains. Previous studies across multiple diverse samples have shown that experiential avoidance is related to poorer health outcomes, higher rates of mental health diagnoses, and worse emotional functioning (Costa \& Pinto-Gouveia, 2013; Masuda, Muto, Tully, Morgan, \& Hill, 2014; Spinhoven et al., 2014). Yet, few studies have examined measurement invariance between racial/ethnic groups casting some doubt upon the assessment accuracy of experiential avoidance among these groups. The current findings, however, provide preliminary support for the use and comparison of the AAQ-II one factor model as a measurement of experiential avoidance between H/L and NHW undergraduates. In addition, the current findings support previous research assessing measurement invariance of the AAQ-II, which have found the one factor model to be 
invariant across samples from various countries (United Kingdom, Italy, Greece, Spain, France, Colombia) speaking various languages (Greek, French, Spanish, Italian; Eisenbeck \& SzabóBartha, 2018; Karekla \& Michaelides, 2017; Ruiz et al., 2016; Zhang et al., 2014).

Across NHW and H/L individuals, higher experiential avoidance was associated with worse scores on important psychological processes and functioning measures. However, two differences in correlational patterns emerged between racial/ethnic groups. First, experiential avoidance was more strongly related to worse anxiety for NHW individuals as compared to H/L individuals. Second, experiential avoidance was weakly associated with polysubstance use (i.e. number of substances used in the past month) for NHW individuals, but had no significant association among $\mathrm{H} / \mathrm{L}$ individuals. Given that the current findings support score comparisons between racial/ethnic groups, the statistically significant differences in correlation coefficients suggest that experiential avoidance may differentially relate to anxiety and polysubstance use among NHW and H/L undergraduates. The current study lacks the ability to speculate on possible influential factors, and given possible conflation between the AAQ-II and measures of distress, these correlations should be interpreted with caution. That said, these findings may suggest that $\mathrm{H} / \mathrm{L}$ students may possess protective factors that serve to buffer some effects of experiential avoidance on polysubstance use and anxiety. Unfortunately, few studies have examined influential factors on experiential avoidance, and even fewer have studied these factors among ethnic and racial minorities. There is a large literature examining protective factors among H/L undergraduates, which include familism, positive ethnic identity, and spirituality. All of which have been associated with less hazardous substance use and improved psychological well-being (Archuleta, 2015; Chun, Marin, Schwartz, Pham, \& Castro-Olivo, 2016; Corona et al., 2017; DiBello, Gonzales, Young, Rodriguez, \& Neighbors, 2016; Morgan Consoli, Delucio, 
Noriega, \& Llamas, 2015). Future research should examine these and other cultural factors in relation to experiential avoidance to better characterize differences found in experiential avoidance between NHW and H/L students.

The current study has a number of limitations. First, the findings may have limited generalizability to other Hispanic/Latinx samples as well as larger undergraduate samples, given that the current sample were primarily undergraduates in introductory psychology courses. Second, measures of language spoken, level of acculturation, country of origin, or generational status were not assessed in the current sample, limiting the ability to discuss these findings in the context of Hispanic/Latinx culture or further elucidate measurement invariance in regard to these variables. The current sample was not seeking clinical services, therefore the findings may also not generalize well to clinical samples. Lastly, given recent attention to the AAQ-II's possible limited discriminant validity between experiential avoidance and the presence of distress as well as limited sensitivity of the measure in non-clinical samples, the correlational findings presented in the current study may be inflated and should be interpreted with caution (Ong, Pierce, Woods, Twohig, \& Levin, 2019; Tyndall et al., 2019; Wolgast, 2014).

Overall, the AAQ-II had strong psychometric properties within the current sample and provides the foundational work needed to better understand experiential avoidance as a transdiagnostic process by ensuring measurement invariance between $\mathrm{H} / \mathrm{L}$ and NHW undergraduates. In addition, validation of the AAQ-II's psychometric properties warrants further study into specific protective and risk factors that may influence experiential avoidance and associated mental health outcomes among H/L individuals. Specifically, examination of cultural values and minority stress factors in relation to experiential avoidance may further highlight the role of experiential avoidance across cultures. Future research should also continue conducting 
tests of measurement invariance across a variety of racial/ethnic groups to increase the applicability of the AAQ-II to a wider range of demographic and cultural communities, as well as to further investigate experiential avoidance as a transdiagnostic process. 


\section{References}

Archuleta, A. J. (2015). Newcomers: The Contribution of Social and Psychological Well-Being on Emotion Regulation Among First-Generation Acculturating Latino Youth in the Southern United States. Child and Adolescent Social Work Journal, 32(3), 281-290. https://doi.org/10.1007/s10560-014-0370-4

Bond, F. W., Hayes, S. C., Baer, R. A., Carpenter, K. M., Guenole, N., Orcutt, H. K., ... Zettle, R. D. (2011). Preliminary psychometric properties of the Acceptance and Action Questionnaire-II: A revised measure of psychological inflexibility and experiential avoidance. Behavior Therapy, 42(4), 676-688. https://doi.org/10.1016/j.beth.2011.03.007

Brazier, J. E., Harper, R., Jones, N. M., O’Cathain, A., Thomas, K. J., Usherwood, T., \& Westlake, L. (1992). Validating the SF-36 health survey questionnaire: New outcome measure for primary care. BMJ (Clinical Research Ed.), 305(6846), 160-164. https://doi.org/10.1136/bmj.305.6846.160

Brown, K. W., \& Ryan, R. M. (2003). The benefits of being present: Mindfulness and its role in psychological well-being. Journal of Personality and Social Psychology, 84(4), 822-848. https://doi.org/10.1037/0022-3514.84.4.822

Camacho, Á., Cordero, E. D., \& Perkins, T. (2016). Psychometric properties of the DASS-21 among Latina/o college students by the US-Mexico border. Journal of Immigrant and Minority Health, 18(5), 1017-1023. https://doi.org/10.1007/s10903-016-0415-1

Chen, F. F., Sousa, K. H., \& West, S. G. (2014). Teacher's corner: Testing measurement invariance of second-order factor models. Structural Equation Modeling, 12(June), 471492. https://doi.org/10.1207/s15328007sem1203

Chun, H., Marin, M. R., Schwartz, J. R., Pham, A., \& Castro-Olivo, S. M. (2016). 
Psychosociocultural Structural Model of College Success among Latina/o Students in Hispanic-Serving Institutions. Journal of Diversity in Higher Education, 9(4), 385-400. https://doi.org/10.1037/a0039881

Cole, D. A., Ciesla, J. A., \& Steiger, J. H. (2007). The insidious effects of failing to include design-driven correlated residuals in latent-variable covariance structure analysis. Psychological Methods, 12(4), 381-398. https://doi.org/10.1037/1082-989X.12.4.381

Corona, R., Rodríguez, V. M., McDonald, S. E., Velazquez, E., Rodríguez, A., \& Fuentes, V. E. (2017). Associations between Cultural Stressors, Cultural Values, and Latina/o College Students' Mental Health. Journal of Youth and Adolescence, 46(1), 63-77. https://doi.org/10.1007/s10964-016-0600-5

Costa, J., \& Pinto-Gouveia, J. (2013). Experiential avoidance and self-compassion in chronic pain. Journal of Applied Social Psychology, 43(8), 1578-1591. https://doi.org/10.1111/jasp.12107

DeBerard, M. S., \& Masters, K. S. (2014). Psychosocial correlates of the Short-Form-36 multidimensional health survey in university students. Psychology, 5, 941-949.

DiBello, A. M., Gonzales, R., Young, C. M., Rodriguez, L. M., \& Neighbors, C. (2016). Blood is thicker than booze: Examining the role of familism and gender in alcohol use and related consequences among Hispanic college students. Journal of Ethnicity in Substance Abuse, 15(3), 310-324. https://doi.org/10.1080/15332640.2015.1044684

Edwards, K. A., Witkiewitz, K., \& Vowles, K. E. (2019). Demographic differences in perceived social norms of drug and alcohol use among Hispanic/Latinx and non-Hispanic White college students. Addictive Behaviors, 98(July), 1-7.

https://doi.org/10.1016/j.addbeh.2019.106060 
Eisenbeck, N., \& Szabó-Bartha, A. (2018). Validation of the Hungarian version of the Acceptance and Action Questionnaire-II (AAQ-II). Journal of Contextual Behavioral Science, 9(February), 80-87. https://doi.org/10.1016/j.jcbs.2018.07.007

Fledderus, M., Voshaar, M. A. H. O., Klooster, P. M., \& Bohlmeijer, E. T. (2012). Further evaluation of the psychometric properties of the Acceptance and Action Questionnaire - II. Psychological Assessment, 24(4), 925-936. https://doi.org/10.1037/a0028200

Flynn, M. K., Berkout, O. V., \& Bordieri, M. J. (2016). Cultural considerations in the measurement of psychological flexibility: Initial validation of the Acceptance and Action Questionnaire-II among Hispanic individuals. Behavior Analysis: Research and Practice, 16(2), 81-93. https://doi.org/10.1037/bar0000035

Gámez, W., Chmielewski, M., Kotov, R., Ruggero, C., Suzuki, N., \& Watson, D. (2014). The brief experiential avoidance questionnaire: Development and initial validation. Psychological Assessment, 26(1), 35-45. https://doi.org/10.1037/a0034473

Hayes, S. C., Pistorello, J., \& Levin, M. E. (2012). Acceptance and commitment therapy as a unified model of behavior change. The Counseling Psychologist, 40(7), 976-1002. https://doi.org/10.1177/0011000012460836

Hayes, S. C., Wilson, K. G., Gifford, E. V, Follette, V. M., \& Strosahl, K. D. (1996). Experiential avoidance and behavioral disorders: A functional dimensional approach to diagnosis and treatment. Journal of Consulting and Clinical Psychology, 64(6), 1152-1168.

Karekla, M., \& Michaelides, M. P. (2017). Validation and invariance testing of the Greek adaptation of the Acceptance and Action Questionnaire -II across clinical vs. nonclinical samples and sexes. Journal of Contextual Behavioral Science, 6(1), 119-124. https://doi.org/10.1016/j.jcbs.2016.11.006 
Kashdan, T. B., Barrios, V., Forsyth, J. P., \& Steger, M. F. (2006). Experiential avoidance as a generalized psychological vulnerability: Comparisons with coping and emotion regulation strategies. Behaviour Research and Therapy, 44(9), 1301-1320. https://doi.org/10.1016/j.brat.2005.10.003

Katerndahl, D. A., Amodei, N., Larme, A. C., \& Palmer, R. (2002). Psychometric assessment of measures of patients in a primary care setting. Psychological Reports, 91, 1121-1128.

Kline, R. B. (2015). Principles and practice of structural equation modeling. (T. D. Little, Ed.) (Fourth Edi). New York, NY: Guildford Publications, Inc.

Levin, M. E., MacLane, C., Daflos, S., Seeley, J. R., Hayes, S. C., Biglan, A., \& Pistorello, J. (2013). Examining psychological inflexibility as a transdiagnostic process across psychological disorders. Journal of Contextual Behavioral Science, 3(3), 155-163. https://doi.org/10.1016/j.jcbs.2014.06.003

Li, C. H. (2016). Confirmatory factor analysis with ordinal data: Comparing robust maximum likelihood and diagonally weighted least squares. Behavior Research Methods, 48(3), 936949. https://doi.org/10.3758/s13428-015-0619-7

Masuda, A., Muto, T., Tully, E. C., Morgan, J., \& Hill, M. L. (2014). Comparing Japanese college students' and U.S. college students' disordered eating, distress, and psychological inflexibility. Journal of Cross-Cultural Psychology, 45(7), 1162-1174. https://doi.org/10.1177/0022022114534982

McCracken, L. M., \& Vowles, K. E. (2014). Acceptance and commitment therapy and mindfulness for chronic pain: Model, process, and progress. American Psychologist, 69(2), 178-187. https://doi.org/10.1037/a0035623

Monestès, J. L., Karekla, M., Jacobs, N., Michaelides, M. P., Hooper, N., Kleen, M., ... Hayes, 
S. C. (2018). Experiential avoidance as a common psychological process in European cultures. European Journal of Psychological Assessment, 34(4), 247-257. https://doi.org/10.1027/1015-5759/a000327

Morgan Consoli, M. L., Delucio, K., Noriega, E., \& Llamas, J. (2015). Predictors of Resilience and Thriving Among Latina/o Undergraduate Students. Hispanic Journal of Behavioral Sciences, 37(3), 304-318. https://doi.org/10.1177/0739986315589141

Muthén, L. K., \& Muthén, B. O. (2017). Mplus User's Guide (Eigth Edit). Los Angeles, CA: Muthén \& Muthén.

Neff, K. D. (2003). The development and validation of a scale to measure self-compassion. Self and Identity, 2, 223-250. https://doi.org/10.1080/15298860390209035

Neff, K. D., Tóth-Király, I., Yarnell, L. M., Arimitsu, K., Castilho, P., Ghorbani, N., ... Mantzios, M. (2018). Examining the factor structure of the Self-Compassion Scale in 20 diverse samples: Support for use of a total score and six subscale scores. Psychological Assessment, 31(1), 27-45. https://doi.org/10.1037/pas0000629

Neff, K. D., Whittaker, T. A., \& Karl, A. (2017). Examining the factor structure of the SelfCompassion Scale in four distinct populations: Is the use of a total scale score justified? Journal of Personality Assessment, 99(6), 596-607. https://doi.org/10.1080/00223891.2016.1269334

Ong, C. W., Pierce, B. G., Woods, D. W., Twohig, M. P., \& Levin, M. E. (2019). The Acceptance and Action Questionnaire - II: An item response theory analysis. Journal of Psychopathology and Behavioral Assessment, 41, 123-134. https://doi.org/10.1016/S00225347(06)00274-6

Osman, A., Lamis, D. A., Bagge, C. L., Freedenthal, S., Barnes, S. M., Osman, A., ... 
Freedenthal, S. (2016). The Mindful Attention Awareness Scale: Further examination of dimensionality, reliability, and concurrent validity estimates. Journal of Personality Assessment, 98(2), 189-199. https://doi.org/10.1080/00223891.2015.1095761

Osman, A., Wong, J. L., Bagge, C. L., Freedenthal, S., Gutierrez, P. M., \& Lozano, G. (2012). The Depression Anxiety Stress Scales-21 (DASS-21): Further examination of fimensions, scale, reliability, and correlates. Journal of Clinical Psychology, 68(12), 1322-1338. https://doi.org/10.1002/jclp.21908

Rolffs, J. L., Rogge, R. D., \& Wilson, K. G. (2016). Disentangling components of flexibility via the hexaflex model. Assessment, 107319111664590. https://doi.org/10.1177/1073191116645905

Ruiz, F. J., Suárez-Falcón, J. C., Cárdenas-Sierra, S., Durán, Y., Guerrero, K., \& RiañoHernández, D. (2016). Psychometric properties of the Acceptance and Action Questionnaire-II in Colombia. Psychological Record, 66(3), 429-437. https://doi.org/10.1007/s40732-016-0183-2

Shorey, R. C., Gawrysiak, M. J., Elmquist, J., Brem, M., Anderson, S., \& Stuart, G. L. (2017). Experiential avoidance, distress tolerance, and substance use cravings among adults in residential treatment for substance use disorders. Journal of Addictive Diseases, 36(3), 151157. https://doi.org/10.1080/10550887.2017.1302661

Spinhoven, P., Drost, J., de Rooij, M., van Hemert, A. M., \& Penninx, B. W. (2014). A longitudinal study of experiential avoidance in emotional disorders. Behavior Therapy, 45(6), 840-850. https://doi.org/10.1016/j.beth.2014.07.001

Stockton, D., Kellett, S., Berrios, R., Sirois, F., Wilkinson, N., \& Miles, G. (2018). Identifying the underlying mechanisms of change during acceptance and commitment therapy (ACT): 
A systematic review of contemporary mediation studies. Behavioural and Cognitive Psychotherapy, (October 2018), 1-31. https://doi.org/10.1017/S1352465818000553

Su, J. C., Wei, M., \& Tsai, H. T. (2014). Running away from unwanted feelings: Culture matters. Cognition and Emotion, 28(7), 1313-1327. https://doi.org/10.1080/02699931.2014.881322 Tyndall, I., Waldeck, D., Pancani, L., Whelan, R., Roche, B., \& Dawson, D. L. (2019). The Acceptance and Action Questionnaire-II (AAQ-II) as a measure of experiential avoidance: Concerns over discriminant validity. Journal of Contextual Behavioral Science, 12, 278284. https://doi.org/10.1016/j.jcbs.2018.09.005

Wolgast, M. (2014). What does the acceptance and action questionnaire (AAQ-II) really measure? Behavior Therapy, 45(6), 831-839. https://doi.org/10.1016/j.beth.2014.07.002

Zhang, C. Q., Chung, P. K., Si, G., \& Liu, J. D. (2014). Psychometric properties of the acceptance and action questionnaire-II for Chinese college students and elite Chinese athletes. Measurement and Evaluation in Counseling and Development, 47(4), 256-270. https://doi.org/10.1177/0748175614538064

Zvolensky, M. J., Jardin, C., Garey, L., Robles, Z., \& Sharp, C. (2016). Acculturative stress and experiential avoidance: relations to depression, suicide, and anxiety symptoms among minority college students. Cognitive Behaviour Therapy, 45(6), 501-517. https://doi.org/10.1080/16506073.2016.1205658 